\title{
Visual loss in HIV-associated cryptococcal meningitis: A case series and review of the mechanisms involved
}

\begin{tabular}{|c|c|}
\hline \multicolumn{2}{|c|}{$\begin{array}{l}\text { Authors: } \\
\text { Anand Moodley }{ }^{1,2} \\
\text { William Rae }^{3} \\
\text { Ahmed Bhigjee }^{2}\end{array}$} \\
\hline \multicolumn{2}{|c|}{$\begin{array}{l}\text { Affiliations: } \\
\text { 'Department of Neurology, } \\
\text { Greys Hospital, South Africa }\end{array}$} \\
\hline \multicolumn{2}{|c|}{$\begin{array}{l}\text { Department of Neurology, } \\
\text { University of KwaZulu-Natal, } \\
\text { South Africa }\end{array}$} \\
\hline \multicolumn{2}{|c|}{$\begin{array}{l}{ }^{3} \text { Department of Medical } \\
\text { Physics, University of The } \\
\text { Free State, South Africa }\end{array}$} \\
\hline \multicolumn{2}{|c|}{$\begin{array}{l}\text { Correspondence to: } \\
\text { Anand Moodley }\end{array}$} \\
\hline \multicolumn{2}{|c|}{$\begin{array}{l}\text { Email: } \\
\text { anand.moodley1@gmail.com }\end{array}$} \\
\hline \multicolumn{2}{|c|}{$\begin{array}{l}\text { Postal address: } \\
\text { PO Box 13833, Casca } \\
\text { 3202, South Africa }\end{array}$} \\
\hline \multicolumn{2}{|c|}{$\begin{array}{l}\text { Dates: } \\
\text { Received: } 17 \text { Dec. } 2014 \\
\text { Accepted: } 21 \text { Aug. } 2015 \\
\text { Published: } 16 \text { Oct. } 2015\end{array}$} \\
\hline \multicolumn{2}{|c|}{$\begin{array}{l}\text { How to cite this article: } \\
\text { Moodley A, Rae W, Bhigjee A. } \\
\text { Visual loss in HIV-associated } \\
\text { cryptococcal meningitis: } \\
\text { A case series and review of } \\
\text { the mechanisms involved. } \\
\text { S Afr J HIV Med. } 2015 ; 16(1) \text {, } \\
\text { Art. \#305, } 9 \text { pages. http:// } \\
\text { dx.doi.org/10.4102/ } \\
\text { sajhivmed.v16i1.305 }\end{array}$} \\
\hline \multicolumn{2}{|c|}{$\begin{array}{l}\text { Copyright: } \\
\text { C 2015. The Authors. } \\
\text { Licensee: AOSIS } \\
\text { OpenJournals. This work is } \\
\text { licensed under the Creative } \\
\text { Commons Attribution } \\
\text { License. }\end{array}$} \\
\hline \multicolumn{2}{|l|}{ Read online: } \\
\hline 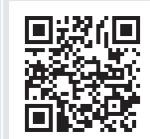 & $\begin{array}{l}\text { Scan this QR } \\
\text { code with your } \\
\text { smart phone or } \\
\text { mobile device } \\
\text { to read online. }\end{array}$ \\
\hline
\end{tabular}

Permanent visual loss is a devastating yet preventable complication of cryptococcal meningitis. Early and aggressive management of cerebrospinal fluid pressure in conjunction with antifungal therapy is required. Historically, the mechanisms of visual loss in cryptococcal meningitis have included optic neuritis and papilloedema. Hence, the basis of visual loss therapy has been steroid therapy and intracranial pressure lowering without clear guidelines. With the use of high-resolution magnetic resonance imaging of the optic nerve, an additional mechanism has emerged, namely an optic nerve sheath compartment syndrome (ONSCS) caused by severely elevated intracranial pressure and fungal loading in the peri-optic space. An improved understanding of these mechanisms and recognition of the important role played by raised intracranial pressure allows for more targeted treatment measures and better outcomes. In the present case series of $90 \mathrm{HIV}$ co-infected patients with cryptococcal meningitis, we present the clinical and electrophysiological manifestations of Cryptococcusinduced visual loss and review the mechanisms involved.

\section{Introduction}

Meningitis owing to Cryptococcus neoformans remains a frequent human immunodeficiency virus (HIV)- associated opportunistic infection even in developing countries with effective antiretroviral therapy (ART) rollout programmes. ${ }^{1}$ This is largely a result of failure of HIV testing by individuals with risky sexual behaviour, and late presentation for and poor compliance with ART. Therefore, it is not uncommon to still encounter severely immunocompromised patients presenting for the first time with opportunistic infections and CD4+ T-lymphocyte counts $<100$ cells $/ \mu$ L. Headache, high fever, nuchal pain and stiffness, photophobia, confusion, nausea, vomiting and diplopia are the common presenting symptoms of cryptococcal meningitis (CM). Symptoms arise from raised intracranial pressure and meningeal inflammation, usually within 1-2 weeks of the onset of the illness. High cerebrospinal fluid (CSF) pressure, depressed level of consciousness and an acellular CSF are poor prognostic features. Effective antifungal therapy (amphotericin B, flucytosine and fluconazole) is not readily available in most developing countries. ${ }^{2}$ Mortality remains high and contributes up to $20 \%$ of HIV-related deaths. ${ }^{1}$ Complications in survivors are severe, with visual loss being the most disabling, yet are potentially preventable and reversible. Recognition of visual impairment in encephalopathic patients is difficult and therefore often neglected and underreported. In the following case series, we evaluated 90 patients with culture-confirmed CM. Their results and a discussion of the mechanisms implicated in Cryptococcus-induced visual loss are discussed. An illustrative case of the optic nerve sheath compartment syndrome (ONSCS) as a putative mechanism is also presented in the discussion.

\section{Method}

In a prospective study approved by the Greys Hospital and University of KwaZulu-Natal Ethics Committees, we consecutively recruited 90 patients with culture-confirmed CM between February 2008 and December 2011 (Table 1). Patients with reduced levels of consciousness were excluded (GCS < 14). All were HIV co-infected, provided informed consent, had full neuroophthalmological assessments and had magnetic resonance imaging (MRI) using standard imaging protocols. Patients were recruited within 4 weeks of the disease onset, during the induction and consolidation phases of CM treatment. Drug treatment and management of raised intracranial pressure were based on the 2007 South African HIV Clinician Society Guidelines. ${ }^{3}$

Eighty-six patients underwent electrophysiological testing that involved visual evoked potentials (VEP) and Humphreys visual fields (HVF). VEP involved testing of each optic nerve's functioning by requesting the patient to look at a screen one metre away that displayed an alternating full-field checkerboard pattern. The cortical responses thus obtained were detected by silver-surface electrodes placed over the occipital scalp. Averaging of the cortical responses provided a reliable and reproducible triphasic wave from which the P100 latency (the large 
TABLE 1: Demographic data, cerebrospinal fluid pressure and CD4 count of 90 cryptococcal meningitis patients.

\begin{tabular}{ll}
\hline Patient characteristics & Patient data \\
\hline Age in years: mean (range) & $33.5(18-51)$ \\
Male: $n(\%)$ & $50(55.6 \%)$ \\
CD4 count in cells/ $\mu$ L: mean (s.d.) & $47(10.1)$ \\
On ART: $n(\%)$ & $22 / 90(24.4 \%)$ \\
CSF pressure in Cm CSF: mean (s.d.) & $31.3(13.5)$ \\
\hline
\end{tabular}

s.d., standard deviation; ART, antiretroviral therapy; CSF, cerebrospinal fluid.

positive wave that occurs at approximately $100 \mathrm{~ms}$ from the stimulus) and amplitude (the vertical height in $\mu \mathrm{V}$ between the largest positive P100 and negative N80 waves) were obtained in accordance with International Society for Clinical Electrophysiology of Vision (ISCEV) guidelines. ${ }^{4}$ HVF was performed using the SITA 30-2 standard protocol. Pattern deviation fields that fulfilled acceptable reliability indices were included for analysis. Flash VEP using LED goggles were used in patients who were delirious; however, HVF was not possible in such patients.

\section{Statistics}

Visual acuity, VEP latency and amplitude were dichotomised into abnormal and normal groups using standard normal references. One-sample $t$ tests were used to compare mean latency and amplitude with laboratory references that have been previously described. ${ }^{5}$ Tests for association between groups were analysed using a chi-square test or Fisher's exact test, as appropriate. Statistical analysis was done by STATA, version 12 .

\section{Results and discussion Clinical findings}

Visual loss occurred at any stage of the illness and occurred frequently before starting drug therapy. Subgroup analysis not reflected in Table 2 showed that the majority of cases occurred within 2-4 weeks of CM onset, regardless of drug therapy. Rex's landmark article in 1993 of Cryptococcusinduced visual loss suggested two main mechanisms: an early and sudden visual loss owing to optic neuritis, and a late and gradual visual loss owing to papilloedema. Such distinct mechanisms, however, do not exist in isolation and an explanation for visual loss where neither mechanism is in operation needs clarification. ${ }^{6}$ Gradual, symmetrical and bilateral visual blurring associated with headache was the most common presentation in our series (Table 2). Sudden and catastrophic visual loss was rare, occurring in only one patient. Forty-six percent of patients had appreciable loss of vision $(<6 / 9$ on Snellen) and profound visual loss of $<6 / 60$ in $13 \%$. Colour desaturation, pupillary reflex changes and pain on eye movement were relatively uncommon. Sixth nerve palsies owing to elevated intracranial pressure or meningitis occurred in $16 \%$ of patients. Bilateral and symmetrical cerebellar ataxia was common in this group and probably accounted for the impaired smooth pursuit and nystagmus - findings also commonly encountered in HIVassociated neurocognitive disorder.
TABLE 2: Neuro-ophthalmological manifestations of cryptococcal meningitis in 90 patients.

\begin{tabular}{llcc}
\hline $\begin{array}{l}\text { Examination } \\
\text { parameter }\end{array}$ & Clinical findings & \multicolumn{2}{c}{ Proportion examined } \\
\cline { 2 - 4 } & & Proportion & $\%$ \\
\hline Best corrected visual & $<6 / 9$ & $41 / 90$ & 46 \\
acuity (Snellen) & $<6 / 60$ & $12 / 90$ & 13 \\
Mode of onset of & Bilateral/unilateral & $34 / 41 ; 7 / 41$ & $83 ; 17$ \\
visual loss & $<1$ week & $6 / 41$ & 15 \\
& $>1$ week & $35 / 41$ & 85 \\
& Sudden & $1 / 41$ & 2 \\
& Pain on eye movement & $1 / 41$ & 2 \\
& Colour desaturation & $5 / 41$ & 12 \\
External & Bilateral 6th nerve palsy & $7 / 90$ & 8 \\
ophthalmoplegia & Unilateral 6th nerve palsy & $7 / 90$ & 8 \\
Supranuclear eye & Unilateral 3rd nerve palsy & $1 / 90$ & 1 \\
movements & Impaired smooth pursuit & $23 / 90$ & 26 \\
& Gaze-evoked nystagmus & $20 / 90$ & 22 \\
Swollen optic disc & Convergence spasm & $1 / 90$ & 1 \\
\hline Pale optic disc & Unilateral & $26 / 90$ & 29 \\
Pupillary reflex & Bilateral and mild & $3 / 90$ & 3 \\
& Reactive but sluggish & $2 / 90$ & 2 \\
\hline NAPD, & No reaction & $11 / 90$ & 12 \\
& RAPD & $4 / 90$ & 4 \\
\hline
\end{tabular}

RAPD, relative afferent pupillary defect.

\section{Electrophysiological findings}

VEP testing and HVF defects were common in the series we reported, both in visually impaired and visually normal patients with CM (Table 3). In the cross-section of $86 / 90$ patients who underwent electrophysiological tests, VEP abnormalities were detected in visually impaired patients (68.9\% of right eyes and $67.6 \%$ of left eyes), and in visually normal patients (56.5\% of all eyes). In subgroup analysis, prolongation of the P100 latency was the predominant abnormality $\left(42.3 \%\right.$ of all eyes). ${ }^{5}$ In the absence of demyelination, these findings were interpreted as resulting from conduction block caused by optic nerve compression. Optic nerve compression with secondary conduction block and optic nerve infiltration were both deemed likely from these findings. VEP amplitude changes suggesting axonal loss were less frequent (14.6\%) in eyes tested. As shown in Table 3, HVF abnormalities were also very frequent in patients who could be tested $(76.6 \%$ of right eyes and $71.1 \%$ of left eyes). The predominant field defects were peripheral constriction with large blind spots - field defects consistent with papilloedema-related optic nerve dysfunction (Figure 1). ${ }^{5}$ Consequently, the interpretation of these findings was that the HVF defects supported raised intracranial pressure as an important cause of optic nerve dysfunction in Cryptococcusinduced visual loss.

\section{Mechanisms implicated}

Rex's classification of visual loss was time based. ${ }^{6} \mathrm{He}$ suggested that early visual loss was a result of optic nerve infiltration/inflammation and occurred within 6 days of the onset of meningitic symptoms, whereas late visual loss occurred a few weeks into the infection and was the result of optic disc oedema from raised intracranial pressure 
TABLE 3: Frequencies of abnormal visual acuity, visual evoked potentials and Humphreys visual fields in 86 patients tested.

\begin{tabular}{|c|c|c|c|c|c|c|c|c|c|c|}
\hline \multirow[t]{3}{*}{ Findings } & \multirow{2}{*}{\multicolumn{2}{|c|}{$\begin{array}{c}\text { Visual } \\
\text { acuity: }<6 / 9\end{array}$}} & \multicolumn{4}{|c|}{ VEP } & \multicolumn{4}{|c|}{ HVF } \\
\hline & & & \multicolumn{2}{|c|}{ Right eye } & \multicolumn{2}{|c|}{ Left eye } & \multicolumn{2}{|c|}{ Right eye } & \multicolumn{2}{|c|}{ Left eye } \\
\hline & $n$ & $\%$ & $n$ & $\%$ & $n$ & $\%$ & $n$ & $\%$ & $n$ & $\%$ \\
\hline Normal & 46 & 53.5 & 23 & 31.1 & 24 & 32.4 & 11 & 23.4 & 13 & 28.9 \\
\hline Abnormal & 40 & 46.5 & 51 & 68.9 & 50 & 67.6 & 36 & 76.6 & 32 & 71.1 \\
\hline Total & 86 & 100 & 74 & 100 & 74 & 100 & 47 & 100 & 45 & 100 \\
\hline
\end{tabular}

Source: Moodley A, Rae W, Bhigjee A, et al. Early clinical and subclinical visual evoked potential and Humphrey's visual field defects in cryptococcal meningitis. Plos One. 2012;7:e52895. PMID: 23285220, http://dx.doi.org/10.1371/journal.pone.0052895 VEP, visual evoked potential; HVF, Humphrey's visual fieldz

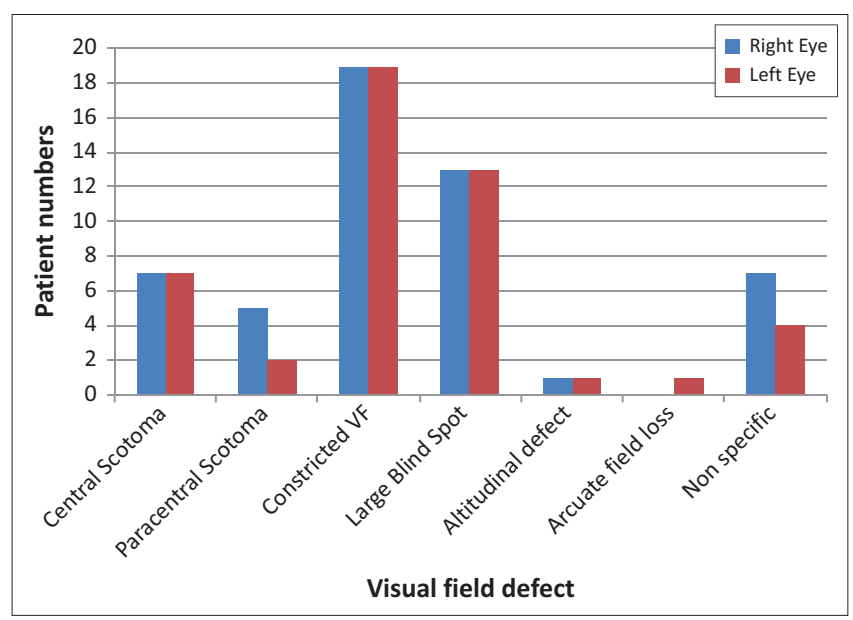

Source: Moodley A, Rae W, Bhigjee A, et al. Early clinical and subclinical visual evoked potential and Humphrey's visual field defects in cryptococcal meningitis. Plos One. 2012;7:e52895. PMID: 23285220, http://dx.doi.org/10.1371/journal.pone.0052895

$\mathrm{VF}$, visual fields.

FIGURE 1: Frequencies of visual field defects.

(papilloedema). Remarkably, the rapid visual loss group in Rex's series had elevated CSF pressure (90\%), thickened optic nerves on computed tomography (CT) scan (22\%) and symmetrical visual loss (93\%). The visual loss occurred before or soon after initiation of antifungal therapy and was severe and permanent. The slow visual loss group did not differ much, having elevated CSF pressure (83\%), thickened optic nerves (22\%) and symmetrical visual loss $(93 \%)$. As to whether there was dilatation of the peri-optic CSF space or thickening of the optic nerve itself was not defined on CT scan for both groups in Rex's series. So, apart from the tempo of presentation, a clear distinction between these groups does seem artificial. We too have previously shown that raised intracranial pressure is common in CMinduced visual loss (69\%), and that papilloedema was present in only $25 \%$; but, in addition, we have shown that on MRI there is no difference between the optic nerve sheath diameter in patients with CM and that of a normal control group, regardless of CSF pressure. ${ }^{7}$ None of the optic nerves demonstrated post-contrast enhancement either, reflecting a poor inflammatory response. Evidence for a third mechanism of optic nerve dysfunction was compelling.

Subsequent reports, as discussed below, have supported or refuted the findings of Rex with evidence for and against the optic neuritis and papilloedema models. However, his work certainly laid down the foundation for investigation into Cryptococcus-induced visual loss; and in fact much of our current understanding has resulted from his original observations.

Recovery of vision has always been documented as poor. Drug treatment alone is insufficient as demonstrated by Graybill et al. where steroids alone were ineffective but serial lumbar punctures and reduction of CSF pressure were more successful. ${ }^{8}$ In Torres's meta-analysis of rapid and slow visual loss cases, the outcome was generally poor when only the underlying $\mathrm{CM}$ was treated and not the raised intracranial pressure. ${ }^{9}$

\section{The papilloedema mechanism}

Raised intracranial pressure in CM is well documented. ${ }^{10,11}$ CSF outflow obstruction caused by plugging of the arachnoid granulations by the organism and/or polysaccharide capsule is postulated to result in the elevated intracranial pressure (Figure 2c). ${ }^{10,12}$ Good support for obstruction at the arachnoid villi has come from Loyse et al. who demonstrated histopathologically that fungal loading (high fungal burden) occurs within the arachnoid villi and is positively correlated with elevated intracranial pressure. ${ }^{12}$ Bicanic et al. have shown that higher fungal burden and higher cryptococcal antigen titres are associated with higher intracranial pressure and have therefore recommended early and aggressive fungicidal treatment with lowering of intracranial pressure by either serial lumbar punctures or lumbar drainage to lower morbidity and mortality in patients with CM. ${ }^{13}$ In 1993, Garrity et al. performed optic nerve sheath fenestrations in two patients with visual loss and papilloedema. ${ }^{14}$ Following the procedure, both patients had improved vision from lowering of intracranial pressure. Cryptococcal organisms were present in the dural sheaths of both patients. At autopsy of one of the patients, patency of the sheath fenestration was still present.

The evidence for visual loss resulting from raised intracranial pressure and papilloedema, and the benefit from CSF pressure lowering either by serial lumbar punctures, $8,10,15,16,17$ acetazolamide, ${ }^{17,18}$ lumbo-peritoneal (LP) shunt, lumbar drain,,$^{18,19,20}$ ventriculo-peritoneal (VP) shunt ${ }^{10,21}$ and optic nerve sheath fenestration ${ }^{14,22}$ are well documented, but unfortunately mostly anecdotal. Comparative studies between surgical lowering of intracranial pressure and drug-only therapy to prevent or reverse visual loss in $\mathrm{CM}$ have not been done. Lowering of the raised intracranial pressure is shown to improve the overall prognosis of $\mathrm{CM}$ and therefore cannot be ethically withheld in a randomised controlled trial. Pharmaceutical approaches alone to control raised intracranial pressure in CM have not been shown to be effective. Surgically invasive methods to decrease intracranial pressure in $\mathrm{CM}$ also carry their own risks such as over drainage, shunt infection, distal catheter migration and need for shunt revision. ${ }^{23} \mathrm{VP}$ shunts are associated with lower risk of shunt obstruction and revision than LP shunts and are therefore recommended when serial lumbar punctures are ineffective or not an option. 
CT and MRI scans show normal ventricular size in most cases of CM despite profoundly elevated CSF pressure. Presumably the equivalent pressures between the intraventricular fluid and the CSF surrounding the brain and the paucity of intraventricular fungal elements prevent ventricular dilatation, unlike tuberculous meningitis where hydrocephalus is often encountered from blockage at the Sylvian aqueduct or foramina of Lushka and Magendie. ${ }^{10}$ Raised intracranial pressure and fungal loading are common and well described in CM patients, but inflammation is minimal if at all, regardless of HIV coinfection. The frequent finding of an acellular CSF in CM despite markedly elevated CSF pressure is a case in point. The significance of raised intracranial pressure cannot be underestimated in visual loss, and perhaps optic disc swelling and optic nerve infiltration/ inflammation are secondary or co-occurrences. Reports of raised intracranial pressure-related visual loss are many in the literature, and the benefit of early lowering of intracranial pressure in reversing blindness in Cryptococcus-induced visual loss is well documented. 15,19,21,24

\section{The optic nerve infiltration/inflammation mechanism}

Evidence for optic nerve infiltration by $C$. neoformans has come from case reports only. Lipson et al. first described two cases of AIDS-associated cryptococcal arachnoiditis resulting in bilateral visual loss secondary to an optic neuropathy (Figure 2b)..$^{25}$ Ofner's claim of optic nerve infiltration in a patient with visual loss and elevated intracranial pressure was not robust. ${ }^{26}$ Histology obtained from the optic nerve sheath showed fungal infiltration with inflammation, but optic nerve infiltration was only presumed. Histological evidence of cryptococcal infiltration of the intracanalicular segment of the optic nerve with associated necrosis was provided by Cohen et al. in $1993^{27}$ and further supported by a histopathological case reported by Corti et al. in 2010. ${ }^{28}$ Corti's case also showed a perineuritis, but in addition showed optic nerve infiltration by the fungus. By inference, Hoepelman $^{29}$ and Seaton ${ }^{30}$ suggested that corticosteroids could only play a beneficial role in Cryptococcus-induced visual loss by reducing the optic nerve inflammation so induced by the organism. Further support for an optic neuritis model has come from De Schacht's report of a 26-year-old CM patient who developed an immune reconstitution illness with bilateral blindness after starting antiretroviral therapy. ${ }^{31}$ Supposedly, the exaggerated optic nerve inflammation secondary to fungal infiltration caused the bilateral blindness. Unfortunately, such case reports in the literature are scanty and the evidence for optic nerve infiltration is mostly speculative. ${ }^{32}$ In our cohort of patients, optic nerve infiltration was uncommon, as evidenced by the lack of nerve signal changes and enhancement on MRI, and the dissimilar magnetic resonance diffusion parameters to that of optic neuritis. ${ }^{7}$ Optic nerve infiltration possibly results from direct cryptococcal invasion from the peri-optic CSF, or perhaps develops from retrograde extension of the meningoencephalitis from the thalamus and other diencephalic structures that seem particularly susceptible to cryptococcal infiltration. The common finding of pseudocysts and dilated Virchow-Robin spaces in these regions supports this assertion.

\section{The optic nerve sheath compartment mechanism}

We have reported a case that demonstrates the strong likelihood of ONSCS which we propose as a probable third mechanism of optic nerve dysfunction (Figure 2d). During elevated CSF pressure, there was loss of the peri-optic CSF signal on T2 MRI and return of the CSF signal following lowering of CSF pressure. ${ }^{33}$ The stasis of contrast-filled CSF at the mid-orbital segment of the optic nerve sheath suggests complete plugging of the peri-optic space by cryptococcal fungal elements. We further postulate that a large pressure gradient resulted from blockage between the significantly elevated intracranial pressure within the intracranial subarachnoid space (SAS) and the pressure of the proximal peri-optic CSF space. An ONSCS thus followed, causing optic nerve compression, axoplasmic stasis and ischaemia. Optic nerve dysfunction ensued with visual blurring and visual loss.

A subsequent case of a 33-year-old HIV-infected patient with bilateral blindness from CM and elevated CSF pressure (>50 cm CSF) also demonstrates this phenomenon. In addition to the blockage within the optic canals bilaterally, dilatation of the peri-optic CSF space ahead of the obstruction is visible on the left side (Figure 3). Notably, this case showed obstruction within the optic canal, unlike the previous case that showed mid-orbital obstruction. After lowering of the CSF pressure, there is return of the CSF to the orbital peri-optic space. We postulate that, following the blockage at the optic canal level, CSF from the orbital peri-optic space is drained by the perioptic lymphatics and hence there is loss of the CSF signal on the T2 high-resolution scan. This is more plausible than loss of the CSF signal prior to CSF pressure lowering being the result of fungal loading alone, as the interval between the two scans was only 11 days and much too soon for all the fungal elements to clear from that space.

Cohen's histological description of intracanalicular necrosis of the optic nerve and obliteration of the intracanalicular peri-optic space by fungal loading provides the only credible histopathological evidence of a clear compartment syndrome in CM-induced visual loss. ${ }^{27}$

Killer et al. have shown by electron microscopy that the perioptic SAS is not occupied by CSF alone but also by a network of trabeculae, septae and pillars comprising fibroblasts and blood vessels. ${ }^{34}$ They provide histological evidence that the peri-optic SAS narrows in the mid-orbital segment where the delicate trabeculae change into broader septae and stout pillars that subdivide the SAS into compartments. The SAS within the intracanalicular segment is extremely narrow and consists of pillars and trabeculae only. Hence the potential sites of blockage to CSF flow are the mid-orbital and the intracanalicular segments of the peri-optic SAS. 


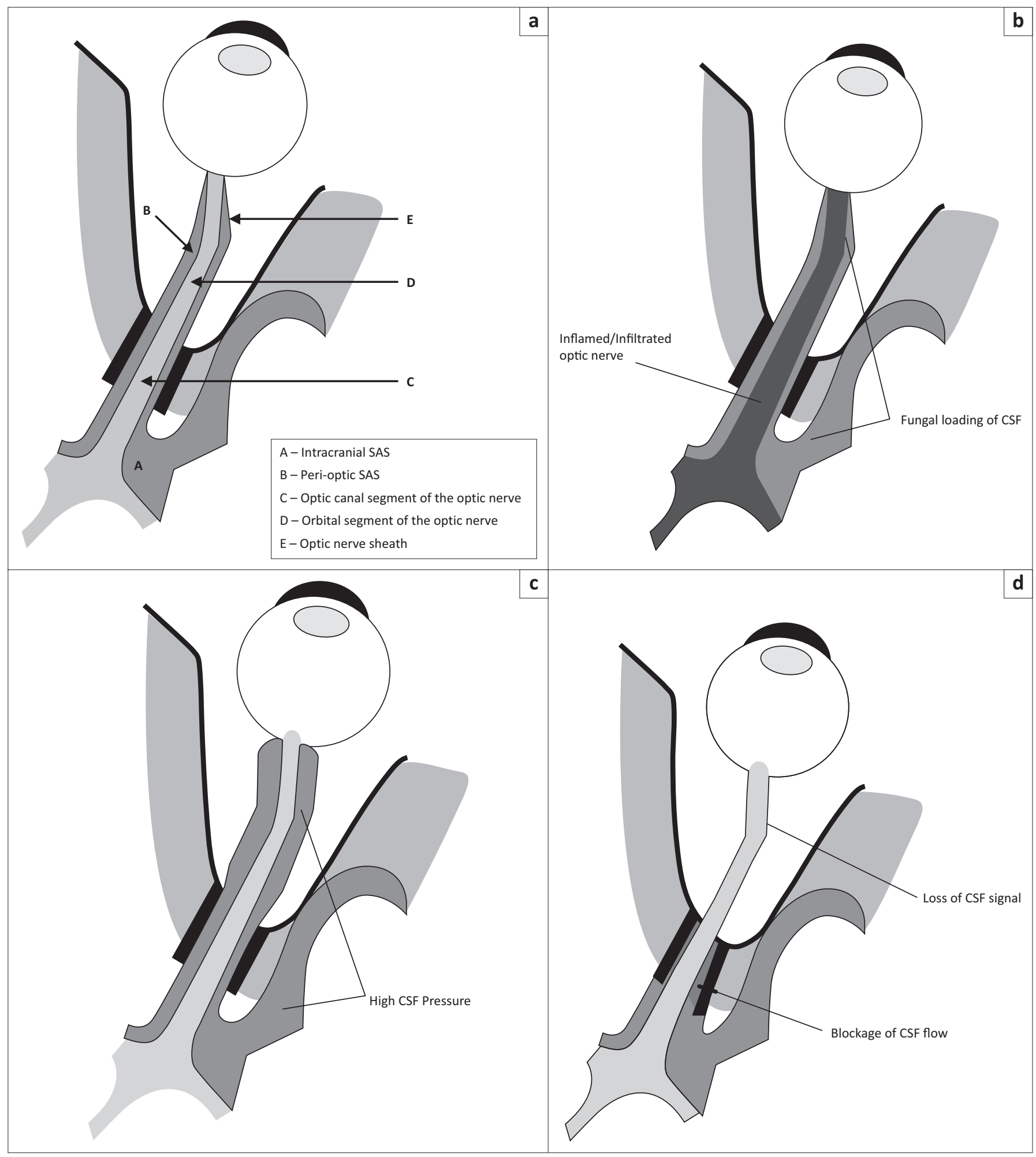

SAS, subarachnoid space; CSF, cerebrospinal fluid.

FIGURE 2: Proposed mechanisms involved in Cryptococcus-induced visual loss. (a) Normal, (b) inflammation/infiltration, (c) papilloedema and (d) compartment syndrome.

Killer et al. also suggest that the varying anatomy of these subarachnoid trabeculae, septae and pillars between the two optic nerves account for the asymmetrical papilloedema in idiopathic intracranial hypertension (IIH). ${ }^{35}$ Asymmetrical pressure is transmitted to the laminar cribrosa of the two optic nerves. On the side with more trabeculae, septae and pillars, a lower pressure is transmitted to the optic nerve head and hence little or no papilloedema ensues. We have expanded on this theory in CM, where, in addition to raised intracranial pressure, there is loading of the peri-optic SAS with fungal elements (the organism and fragments of the polysaccharide capsule). CM is a pauci-inflammatory disorder, and hence fungal loading rather than inflammatory cell accumulation occurs. Fungal loading in the peri-optic CSF space has been shown by histology of the optic nerve sheath during optic nerve sheath fenestration. ${ }^{22}$ Sequestration 
of CSF in the immediate retrobulbar space is not evident, and the contribution made by the fungal clumping needs exploration. We have not been able to demonstrate optic nerve sheath dilatation nor optic nerve thickening in the setting of CM with or without papilloedema and regardless of CSF pressure measured at the lumbar level. ${ }^{7}$ It is conceivable that the retrobulbar segment of the nerve is subjected to toxic byproducts from the fungi, venous stasis, ischaemia from vascular compromise and axoplasmic stasis from mechanical compression. We propose therefore that these findings suggest axoplasmic stasis, mitochondrial dysfunction and ischaemia of the axons which develop from compression at the site of blockage. The end result is optic disc swelling that occurs in only $25 \%$ of CM patients, despite raised intracranial pressure occurring in $69 \%-90 \%$ of CM patients. The blockage at the mid-orbital or intracanalicular segments from raised
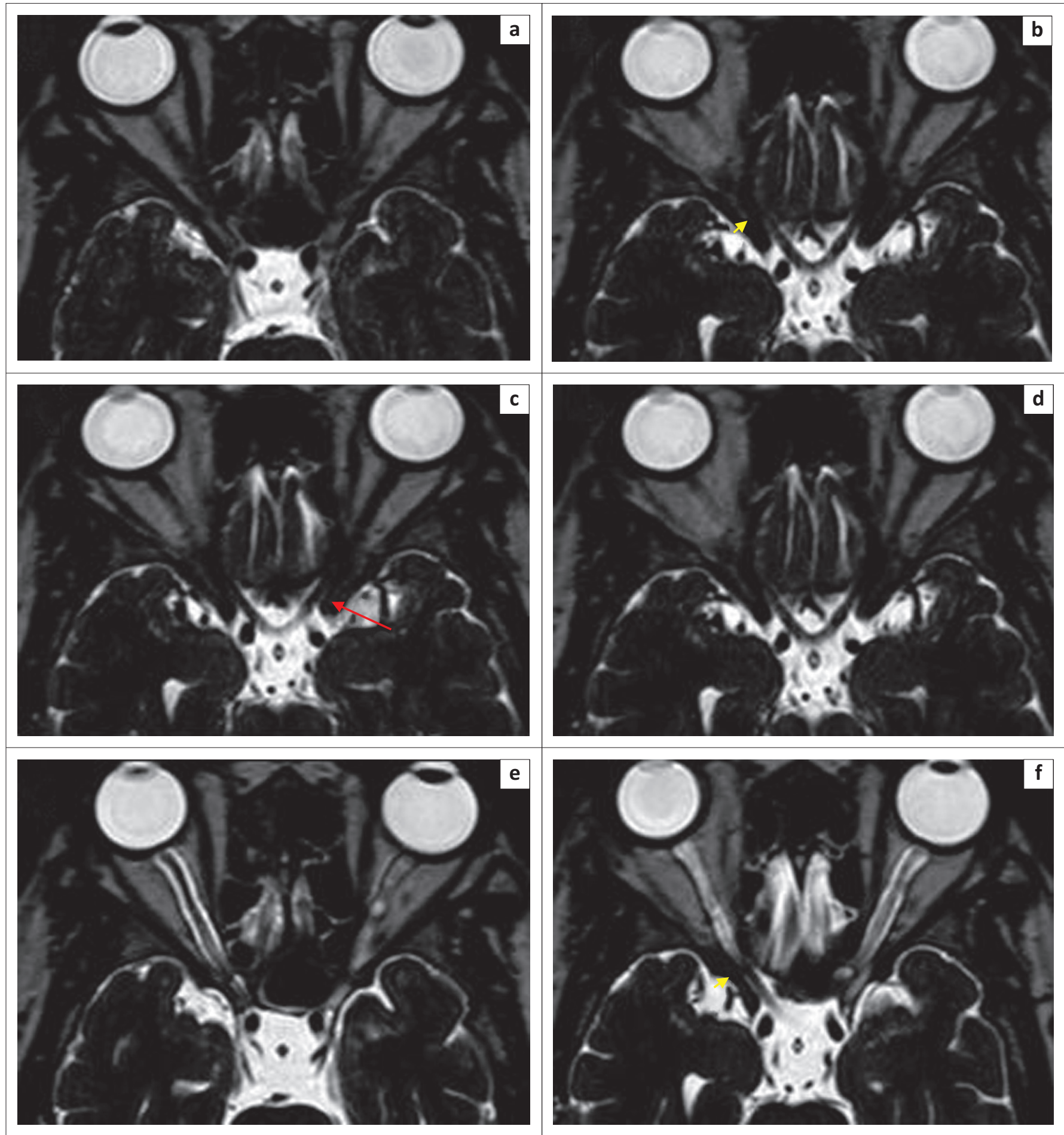

Note: T2 high-resolution magnetic resonance imaging of the optic nerves in a patient with CM and bilateral blindness, showing return of the peri-optic CSF signal following CSF pressure lowering. The red arrows point to the site of blockage within the left optic canal, and yellow arrowheads point to blockage within the optic canal on the right. Re-dilatation of the peri-optic space beyond the
obstruction following pressure lowering is seen on images $\mathrm{e}-\mathrm{h}$.

a-d, Day 1 CSF Pressure $>50 \mathrm{~cm}$ CSF; e-h, Day 11, Following 3 LPs, CSF Pressure $=28 \mathrm{~cm} \mathrm{CSF}$.

FIGURE 3: (a-h), Illustrative case of optic nerve sheath compartment syndrome. 


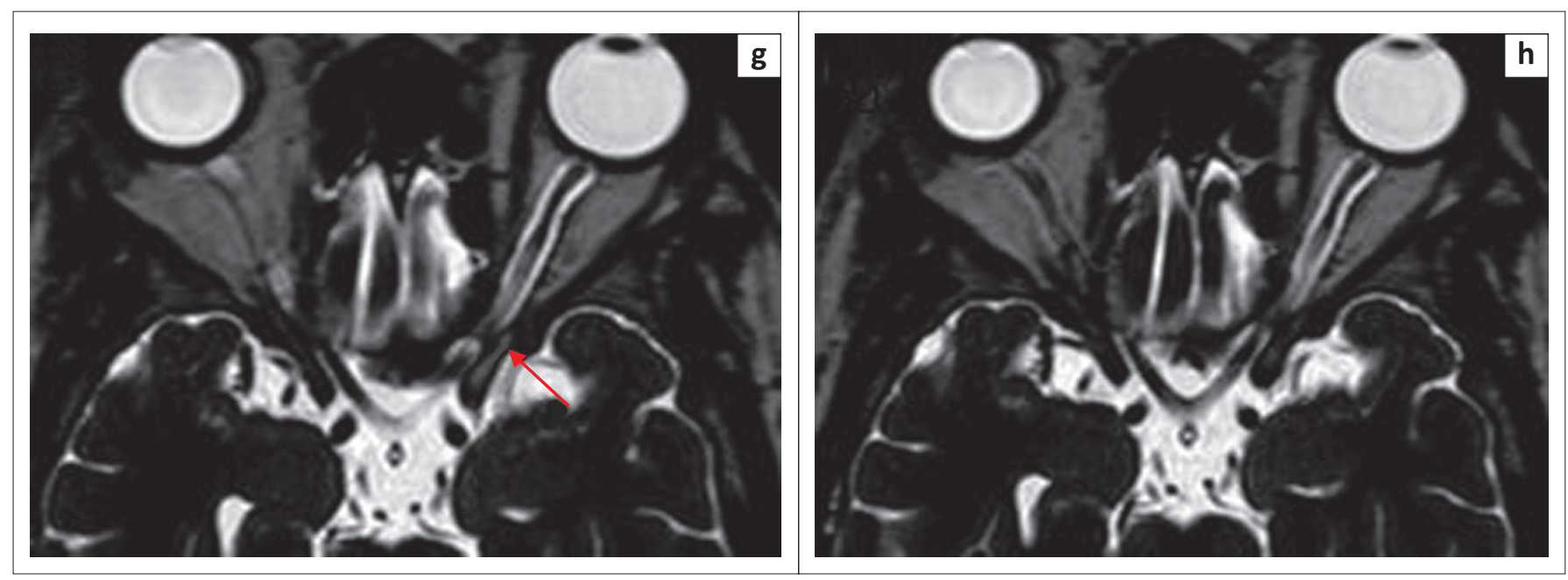

Note: T2 high-resolution magnetic resonance imaging of the optic nerves in a patient with CM and bilateral blindness, showing return of the peri-optic CSF signal following CSF pressure lowering. The red arrows point to the site of blockage within the left optic canal, and yellow arrowheads point to blockage within the optic canal on the right. Re-dilatation of the peri-optic space beyond the obstruction following pressure lowering is seen on images $\mathrm{e}-\mathrm{h}$.

a-d, Day 1 CSF Pressure > $50 \mathrm{~cm}$ CSF; e-h, Day 11, Following 3 LPs, CSF Pressure $=28 \mathrm{~cm} \mathrm{CSF}$.

FIGURE 3 (Continues...): (a-h), Illustrative case of optic nerve sheath compartment syndrome.

pressure and fungal elements creates compartmentalisation between the peri-optic SAS and the intracranial SAS. We propose that the raised pressure and fungal loading cause apposition of the trabeculae, septae and pillars against each other, creating a block by a valve-like mechanism. When intracranial pressure is then lowered, reopening of the channels between the trabeculae and septae occurs, and reestablishment of CSF flow to the peri-optic space.

Furthermore, the lack of optic nerve sheath dilatation and optic nerve signal changes on MRI make papilloedema and optic nerve infiltration less likely to be the only pathogenic mechanisms in CM-induced visual loss. Magnetic resonance diffusion studies do not support optic neuritis as an early cause of visual loss in CM. ${ }^{7}$ The co-occurrence of elevated CSF pressure, swollen optic disc and visual loss was in $15.4 \%$ of our cohort, visual loss and swollen discs in $17.3 \%$, and visual loss and elevated pressure in $26.9 \%$. Whilst visual loss was documented in $34.6 \%$ and elevated pressure was recorded in $69 \%$, clearly disc swelling alone either from optic nerve infiltration or papilloedema was insufficient to account for all cases of visual loss. Elevated CSF pressure with an additional compromise of optic nerve function seems likely - not from axoplasma stasis at the lamina cribrosa but compression upstream. We postulate that this compression results from ONSCS. The steep pressure gradients at the optic canal or mid-orbital level and fungal elements trapped by subarachnoid trabeculae cause a functional block that reverses with pressure lowering. We prefer the term ONSCS to explain the above pathogenesis, which is in line with Killer's explanation of asymmetrical papilloedema in $\mathrm{IIH},{ }^{35}$ but different to the optic nerve compartmentation he described in optic neuritis where CSF was trapped in the bulbar peri-optic space, causing disc swelling. ${ }^{36}$ Our use of the term is also different from Orgul's description of optic nerve compartment syndrome. ${ }^{37}$ Orgul uses the term to describe compartmentalisation in glaucoma where the slitlike pores in the lamina cribrosa cause venous congestion and constriction of the nerve fibre bundles.

\section{Management of visual loss}

Early screening of vision in patients with CM is imperative. Screening should involve proper Snellen chart assessments with pinhole correction if required. Baseline documentation of visual acuity with weekly documentation during the first 4 weeks and bimonthly thereafter until the maintenance phase is complete is essential and should become standard practice. When there is doubt, VEP can be done to detect subtle and even preclinical optic nerve disease. HVF is useful and should be done at initiation of treatment and repeated 4 weeks later when cognition improves with treatment. Field defects are possible, even with intact visual acuity.

With raised intracranial pressure being the predominant mechanism by which visual loss occurs, it is prudent to address this complication in CM. Antifungal therapy alone is insufficient. Early and aggressive lowering of intracranial pressure not only improves the overall prognosis of $\mathrm{CM}$ but also definitely prevents, alleviates and reverses visual loss. ${ }^{13}$ The benefit of intracranial pressure lowering is well documented by using serial lumbar punctures, lumbar drains, VP shunts and optic nerve sheath fenestration. ${ }^{8,10,11,15,16,20,21,22}$ Reports of reversal of visual loss from CSF pressure lowering are encouraging. Medical management alone of raised intracranial pressure in lowering CSF pressure and thereby improving vision has been less satisfactory. ${ }^{38}$ The latest recommendations by the Southern African HIV Clinicians Society (2013) are to remove $10 \mathrm{~mL}-30 \mathrm{~mL}$ of CSF if opening pressure is $>25 \mathrm{~cm} \mathrm{CSF}$ and daily LPs until symptoms of raised intracranial pressure settle. ${ }^{39}$ Evidence for the benefit of corticosteroids and nonsteroidal anti-inflammatory drugs (NSAIDs) to decrease optic nerve inflammation and thus improve vision has been anecdotal at best and is counterintuitive, considering the pauci-inflammatory state of $\mathrm{CM}^{30}$

\section{Conclusion}

The major limitations of our case series have been the lack of long-term follow-up and the exclusion of patients with 


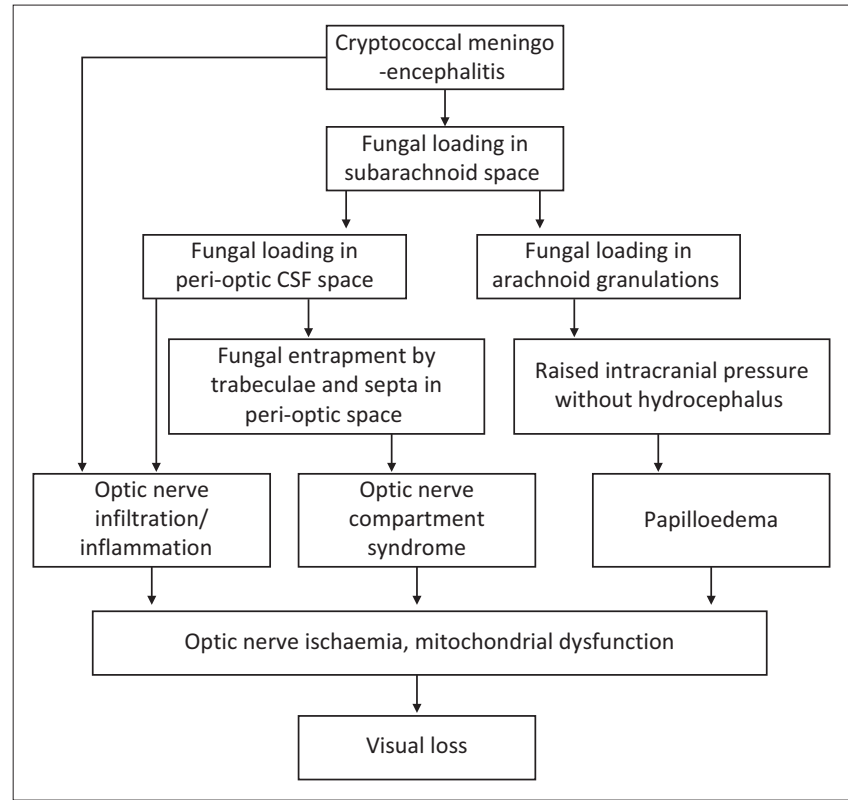

CSF, cerebrospinal fluid.

FIGURE 4: The pathogenesis of Cryptococcus-induced visual loss.

depressed levels of consciousness. However, we feel that the data from this cohort are compelling and certainly contribute to the improved understanding of Cryptococcus-induced visual loss. Visual loss in CM is common and varies from mild loss to no light perception. Bilateral involvement is usual and occurs at any time during the illness, regardless of drug therapy. Electrophysiology shows early and subclinical optic nerve dysfunction in CM. Three mechanisms seem to operate in the pathogenesis of CM-induced visual loss: (1) papilloedema, (2) optic nerve infiltration/inflammation and (3) ONSCS (Figures 2 and 4). Optic nerve infiltration/ inflammation does occur but infrequently and is either a manifestation of the meningo-encephalitis that extends to the optic nerve by continuous spread from the diencephalon or a result of direct infiltration of fungi from the peri-optic CSF space. Raised intracranial pressure plays an important role in visual loss with or without papilloedema. When papilloedema and optic nerve infiltration are not demonstrable, raised intracranial pressure causes optic nerve dysfunction and visual loss, presumably by ONSCS. Fungal loading and obstruction of the peri-optic CSF space compartmentalising the intra-orbital peri-optic SAS from the intracranial SAS is probably the key mechanism but needs further investigation. Our understanding of Cryptococcus-induced visual loss has improved since Rex's initial contribution to the field. However, further studies are eagerly awaited - and preferably those that focus on CSF flow, the peri-optic and intracranial SAS compartments and the impact of pressure lowering measures on visual acuity.

\section{Acknowledgements Competing interests}

The authors declare that they have no financial or personal relationships which may have inappropriately influenced them in writing this article.

\section{Authors' contributions}

A.M. (Greys Hospital) was responsible for drafting of the manuscript, project design, collection and analysis of data. W.R. (University of The Free State) made conceptual contributions and reviewed the manuscript. A.B. (University of KwaZulu-Natal) reviewed the manuscript.

\section{References}

1. Park BJ, Wannemuehler KA, Marston BJ, Govender N, Pappas PG, Chiller TM. Estimation of the current global burden of cryptococcal meningitis among persons
living with HIV/AIDS. AIDS. 2009;23:525-530. PMID: 19182676, http://dx.doi. living with HIV/AIDS. AIDS. 2009;23
org/10.1097/QAD.0b013e328322ffac

2. Loyse A, Thangaraj $H$, Easterbrook $P$, et al. Cryptococcal meningitis: Improving access to essential antifungal medicines in resource-poor countries. Lancet Infect Dis. 2013;13:629-637. PMID: 23735626, http://dx.doi.org/10.1016/S14733099(13)70078-1

3. McCarthy K, Meintjes G. Guidelines for the prevention, diagnosis and management of cryptococcal meningitis and disseminated cryptococcosis in HIV-infected patients: Guideline. S Afr J HIV Med. 2007;28:25-29, 32-35.

4. Odom JV, Bach $M$, Brigell $M$, et al. ISCEV standard for clinical visual evoked potentials (2009 update). Doc Ophthalmol. 2010;120:111-119. PMID: 19826847, http://dx.doi.org/10.1007/s10633-009-9195-4

5. Moodley A, Rae W, Bhigjee A, et al. Early clinical and subclinical visual evoked potential and Humphrey's visual field defects in cryptococcal meningitis. PloS One. 2012;7:e52895. PMID: 23285220, http://dx.doi.org/10.1371/journal.pone. One. 2012

6. Rex JH, Larsen RA, Dismukes WE, Cloud GA, Bennett JE. Catastrophic visual loss due to Cryptococcus neoformans meningitis. Medicine 1993;72:207-224. PMID: 8341139

7. Moodley A, Rae W, Bhigjee A, Loubser N, Michowicz A. New insights into the pathogenesis of Cryptococcal induced visual loss using diffusion-weighted imaging of the optic nerve. Neuro-Ophthalmology. 2012;36:186-192. http://dx.doi.org/10 .3109/01658107.2012.715716

8. Graybill JR, Sobel J, Saag M, et al. Diagnosis and management of increased intracranial pressure in patients with AIDS and cryptococcal meningitis. The NIAID Mycoses Study Group and AIDS Cooperative Treatment Groups. Clin Infect Dis. Mycoses Study Group and AIDS Cooperative Treatment Groups. Clin
2000;30:47-54. PMID: 10619732, http://dx.doi.org/10.1086/313603

9. Torres $\mathrm{OH}$, Negredo $\mathrm{E}$, Ris J, Domingo $P$, Catafau AM. Visual loss due to cryptococcal meningitis in AIDS patients. AIDS. 1999;13:530-532. PMID: 10197388, http:// dx.doi.org/10.1097/00002030-199903110-00018

10. Denning DW, Armstrong RW, Lewis BH, Stevens DA. Elevated cerebrospinal fluid pressures in patients with cryptococcal meningitis and acquired immunodeficiency syndrome. Am J Med. 1991;91:267-272. PMID: 1892147, http://dx.doi. org/10.1016/0002-9343(91)90126-I

11. Johnston SRD, Corbett EL, Foster O, Ash S, Cohen J. Raised intracranial pressure and visual complications in AIDS patients with cryptococcal meningitis. $J$ Infect. 1992;24:185-189. PMID: 1569310, http://dx.doi.org/10.1016/0163-4453(92) 92954-H

12. Loyse $A$, Wainwright $H$, Jarvis $J N$, et al. Histopathology of the arachnoid granulations and brain in HIV-associated cryptococcal meningitis: Correlation with cerebrospinal fluid pressure. AIDS. 2010;24:405-410. PMID: 19952714, http:// cerebrospinal fluid pressure. AlDS. 2010;24:405
dx.doi.org/10.1097/QAD.0b013e328333c005

13. Bicanic T, Brouwer AE, Meintjes $G$, et al. Relationship of cerebrospinal fluid pressure, fungal burden and outcome in patients with cryptococcal meningitis undergoing serial lumbar punctures. AIDS. 2009;23:701-706. PMID: 19279443, undergoing serial lumbar punctures. AlDS. 2009;
http://dx.doi.org/10.1097/QAD.0b013e32832605fe

14. Garrity JA, Herman DC, Imes R, Fries P, Hughes CF, Campbell RJ. Optic nerve sheath decompression for visual loss in patients with acquired immunodeficiency
syndrome and cryptococcal meningitis with papilledema. Am J Ophthalmol. 1993;116:472-478. PMID: 8213978. http://dx.doi.org/10.1016/S0002-9394(14) 71407-2

15. Ferreira RC, Phan G, Bateman JB. Favorable visual outcome in cryptococcal meningitis. Am J Ophthalmol. 1997;124:558-560. PMID: 9323952, http://dx.doi. org/10.1016/S0002-9394(14)70877-3

16. Wijewardana I, Jarvis JN, Meintjes G, Harrison TS, Bicanic T. Large volume lumbar punctures in cryptococcal meningitis clear cryptococcal antigen as well as lowering pressure. J Infect. 2011;63:484-486. PMID: 21930156, http://dx.doi. org/10.1016/j.jinf.2011.09.002

17. Orem J, Tindyebwa L, Twinoweitu O, Mukasa B, Tomberland M, Mbidde EK Feasibility study of serial lumbar puncture and acetazolamide combination in the management of elevated cerebrospinal fluid pressure in AIDS patients with cryptococcal meningitis in Uganda. Trop Doct. 2005;35:19-21. PMID: 15712536 http://dx.doi.org/10.1258/0049475053001967

18. Ng CW, Lam MS, Paton NI. Cryptococcal meningitis resulting in irreversible visual impairment in AIDS patients--a report of two cases. Singapore Med J. 2000;41:8082. PMID: 11063209

19. Claus JJ, Portegies P. Reversible blindness in AIDS-related cryptococcal meningitis. Clin Neurol Neurosurg. 1998;100:51-52. PMID: 9637206, http://dx.doi. org/10.1016/S0303-8467(97)00119-4 
20. Macsween KF, Bicanic T, Brouwer AE, Marsh H, Macallan DC, Harrison TS. Lumbar drainage for control of raised cerebrospinal fluid pressure in cryptococcal drainage for control of raised cerebrospinal fluid pressure in cryptococcal meningitis: Case report and review. J Infect. 2005:
16291274, http://dx.doi.org/10.1016/j.jinf.2005.02.010

21. Petrou P, Moscovici S, Leker RR, Itshayek E, Gomori JM, Cohen JE. Ventriculoperitoneal shunt for intracranial hypertension in cryptococcal 22658489, http://dx.doi.org/10.1016/j.jocn.2012.01.008

22. Milman T, Mirani N, Turbin RE. Optic nerve sheath fenestration in cryptococcal meningitis. Clin Ophthalmol. 2008;2:637-639. PMID: 19668765, http://dx.doi. org/10.2147/OPTH.S2096

23. York J, Bodi I, Reeves I, Riordan-Eva P, Easterbrook PJ. Raised intracranial pressure complicating cryptococcal meningitis: Immune reconstitution inflammatory syndrome or recurrent cryptococcal disease? J Infect. 2005;51:165-171. PMID: 15961162, http://dx.doi.org/10.1016/j.jinf.2005.04.022

24. Chan KH, Mak W, Ho SL. Cryptococcal meningitis with raised intracranial pressure masquerading as malignant hypertension. Int J Infect Dis. 2007;11:366-367. PMID: 17331778, http://dx.doi.org/10.1016/j.ijid.2006.07.005

25. Lipson BK, Freeman WR, Beniz J, et al. Optic neuropathy associated with cryptococcal arachnoiditis in AIDS patients. Am J Ophthalmol. 1989;107:523-527. PMID: 2540660, http://dx.doi.org/10.1016/0002-9394(89)90498-4

26. Ofner S, Baker RS. Visual loss in cryptococcal meningitis. J Clin Neuro-ophthalmol. 1987;7:45-48. PMID: 2952681.

27. Cohen DB, Glasgow BJ. Bilateral optic nerve cryptococcosis in sudden blindness in patients with acquired immune deficiency syndrome. Ophthalmology.
1993;100:1689-1694. PMID: 8233396, http://dx.doi.org/10.1016/S01616420(93)31416-8

28. Corti $M$, Solari R, Cangelosi $D$, et al. Sudden blindness due to bilateral optic neuropathy associated with cryptococcal meningitis in an AIDS patient. Rev Iberoam Micol. 2010;27:207-209. PMID: 20965271, http://dx.doi.org/10.1016/j. riam.2010.09.002

29. Hoepelman Al, Van der Flier M, Coenjaerts FE. Dexamethasone downregulates Cryptococcus neoformans-induced vascular endothelial growth facto production: A role for corticosteroids in cryptococcal meningitis? I Acquir Immune Defic Syndr. 2004;37:1431-1432. PMID: 15483473
30. Seaton RA, Verma N, Naraqi S, Wembri JP, Warrell DA. The effect of corticosteroids on visual loss in Cryptococcus neoformans var. gattii meningitis. Trans R Soc Trop Med Hyg. 1997;91:50-52. PMID: 9093628, http://dx.doi.org/10.1016/S00359203(97)90393-X

31. De Schacht C, Smets RM, Callens S, Colebunders R. Bilateral blindness after starting highly active antiretroviral treatment in a patient with HIV infection and cryptococcal meningitis. Acta Clin Belg. 2005;60:10-12. PMID: 15981698, http:// cryptococcal meningitis. Acta Clin
dx.doi.org/10.1179/acb.2005.003

32. Kestelyn P, Taelman H. Visual loss and cryptococcal meningitis. Trans R Soc Trop Med Hyg. 1997;91:727-728. http://dx.doi.org/10.1016/S0035-9203(97) 90542-3

33. Moodley A, Naidoo N, Reitz D, Chetty N, Rae W. The optic nerve compartment syndrome in Cryptococcus-induced visual loss. Neuro-Ophthalmology. 2013;37:124-128. http://dx.doi.org/10.3109/01658107.2013.792359

34. Killer HE, Laeng HR, Flammer J, Groscurth P. Architecture of arachnoid trabeculae, pillars, and septa in the subarachnoid space of the human optic nerve: Anatomy and clinical considerations. Br J Ophthalmol. 2003;87:777-781. PMID: 12770980 http://dx.doi.org/10.1136/bjo.87.6.777

35. Killer HE, Jaggi GP, Miller NR, et al. Cerebrospinal fluid dynamics between the basal cisterns and the subarachnoid space of the optic nerve in patients with papilloedema. Br J Ophthalmol. 2011;95:822-827. PMID: 20956279, http:// papilloedema. $\mathrm{Br}$ J Ophthalmol. 2011/2010.189324
dx.doi.org/10.1136/bjo.2010.18

36. Killer HE, Mironov A, Flammer J. Optic neuritis with marked distension of the optic nerve sheath due to local fluid congestion. Br J Ophthalmol. 2003;87:249. PMID: 12543769, http://dx.doi.org/10.1136/bjo.87.2.249

37. Orgul S. Compartment syndrome in the optic nerve: A new hypothesis in the pathogenesis of glaucoma. Acta Ophthalmol. 2012:90:686-689. PMID: 21294853 , http://dx.doi.org/10.1111/j.1755-3768.2010.02071.x

38. Megson GM, Stevens DA, Hamilton JR, Denning DW. D-mannitol in cerebrospinal fluid of patients with AIDS and cryptococcal meningitis. J Clin Microbiol. 1996;34:218-221. PMID: 8748311

39. Govender NP, Meintjes G, Bicanic T, et al. Guideline for the prevention, diagnosis and management of cryptococcal meningitis among HIV-infected persons: 2013 update. S Afr J HIV Med. 2013;14:76-86. 